\begin{tabular}{|c|l|}
\hline Title & $\begin{array}{l}\text { Neocortical gap junction-coupled interneuron sy stems may induce chaotic behavior itinerant among quasi-attractors } \\
\text { exhibiting transient synchrony }\end{array}$ \\
\hline Author(s) & Fujii, Hiroshi; Tsuda, Ichiro \\
\hline Citation & $\begin{array}{l}\text { Neurocomputing, 58-60,151-157 } \\
\text { https://doi.org/40.1016j.neucom.2004.01.036 }\end{array}$ \\
\hline Issue Date & 200406 \\
\hline Doc URL & http:/hdl.handle.net/2115/8488 \\
\hline Type & article (author version) \\
\hline File Information & CNSO3_Fujii.pdf \\
\hline
\end{tabular}

Instructions for use 


\title{
Neocortical gap junction-coupled interneuron systems may induce chaotic behavior itinerant among quasi-attractors exhibiting transient synchrony
}

\author{
Hiroshi Fujii* and Ichiro Tsuda \\ Kyoto Sangyo University, Kyoto 603-8555, Japan Hokkaido University, Sapporo 060-0810, Japan
}

\begin{abstract}
Recent discovery of the massive presence of gap junction couplings among neocortical FS (and LTS) interneurons poses serious questions about their collective dynamical behavior, and their possible cognitive roles. We present here the theoretical possibility that a class of neurons coupled by gap junctions may emerge spatio-temporal chaos itinerant among attractors in Milnor's sense, which in turn organizes synchronous cell groups transiently. Some physiological observations from the neocortex, e.g., local field potential (LFP) data exhibiting transient synchrony may provide evidence. We suggest also possible role in the so-called binding problem.
\end{abstract}

Key Words: gap junction; FS interneuron; class I neuron; saddle-node bifurcation; spatio-temporal chaos; chaotic itinerancy; Milnor attractor; transient synchrony; local field potential; binding problem

\section{Introduction}

Recent physiological discovery of ubiquitous presence of gap junctions among FS (and among LTS) interneurons in the neocortex poses serious questions about both their dynamical behavior in in vivo neocortex and their role in cognitive functions. It is generally believed that "a (gap junction-coupled) network of FS cells in the neocortex may play a key role in coordinating cortical activity..." (M. Galarreta \& S. Hestrin, 1999 [5]), or "interneurons generate a variety of

*Corresponding author. fujii@ bacco.kyoto-su.ac.jp (H. Fujii). 
synchronous inhibitory rhythms in the neocortex..." (J. R. Gibson et al., 1999 [6]). Little is known, however, how those interneurons collectively behave when coupled by gap junctions in a massive way.

The purpose of this paper is to present a new result on the collective dynamics of gap junction-coupled neuron systems, based on characterization of nonlinearity of neuron classes [4]. We then discuss possible implications on the behavior of FS cells in the neocortex, suggesting the relation to the observed neocortical LFP (local field potential) fluctuations and transient synchrony [4]. Also, we propose the possibility that the dynamical organization of synchronous cell assemblies by the itinerant chaos may play the role of feature bindings.

\section{Emergent chaos in class I* neurons}

As Hodgkin pointed out in 1948, there exist two classes of "neurons" (actually, "axons"), i.e., class I "axons which are capable of repetition over a wide range of frequencies, varied smoothly over a range of about 5-150 impulses per sec.", and class II "axons which usually give a train of impulses of frequency 75-150/sec which was relatively insensitive to changes in the strength of the applied current" [8]. It is now generally recognized that most of cortical neurons are of class I [1] in the sense of Hodgkin. Mathematically, the difference of behavior between these two classes is attributed to that of generation mechanism of action potentials: saddle-node bifurcations (class I), and subcritical Hopf bifurcations (class II). Although the concept of "class" of neurons does not depend on the number of variables, we restrict our arguments for a moment to a reduced form of two-variables, where the first variable, say V, may represent the membrane potential, and the second one, R, an activation state of, e.g., some potassium channels in a generalized sense [10], [12], [19]. With the injected current strength being denoted by $I$, the single cell equation may be written as:

$$
\left\{\begin{array}{c}
C \frac{d V}{d t}=f(V, R)+I \\
\tau_{R} \frac{d R}{d t}=g(V, R)
\end{array}\right.
$$

If we denote by $\mathrm{N}_{\mathrm{V}}=\left\{(\mathrm{V}, \mathrm{R}) \mid f(V, R)+I=0 \quad\right.$ and $\mathrm{N}_{\mathrm{R}}=\{(\mathrm{V}, \mathrm{R}) \mid g(V, R)=$ 0 \} the nullclines of $\mathrm{V}$ and $\mathrm{R}$, respectively, then class I neurons have generally $\mathrm{J}$ (Morris-Lecar model) or U-shaped (reduced Connor model due Rose-Hindmarsh [ 12 ]) R-nullclines $\mathrm{N}_{\mathrm{R}}$, while class II neurons have inclined I-shaped $\mathrm{N}_{\mathrm{R}}$ ( e.g., FHN model). Since $\mathrm{N}_{\mathrm{V}}$ is essentially cubic, the number of intersections of $\mathrm{N}_{\mathrm{V}}$ and $\mathrm{N}_{\mathrm{R}}$ changes according to the level of $I$, and there appears a saddle-node bifurcation point for Connor neurons, or a homoclinic (saddle-node separatrix loop) bifurcation for the Morris-Lecar model (with appropriately chosen parameters). The salient property of class I neurons of Connor type is that as the injected current $I$ traverses the saddle-node bifurcation point, there appears a 
narrow channel between $\mathrm{N}_{\mathrm{V}}$ and $\mathrm{N}_{\mathrm{R}}$. Rogawski [11] and Rose-Hindmarsh [12] emphasized that this is a consequence of the presence of A-current, i.e., a transient, slowly inactivating potassium current, an ion channel different from the Hodgkin-Huxley's rectifying $\mathrm{K}^{+}$current.

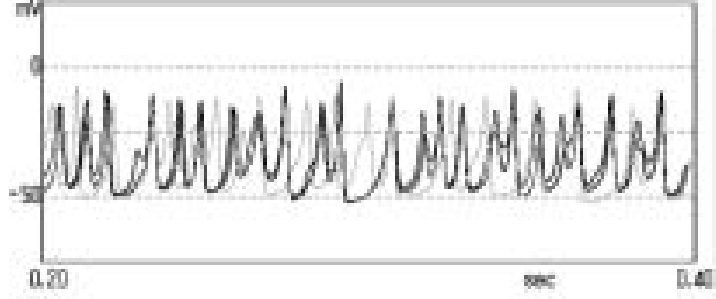

Fig. 1.

Time series of membrane potentials of 3 neurons from $10 x 10$ gap junction-coupled class $I^{*}$ neurons. Coupling is among 4-nearest neighbors.
Our interest is in the study of a system-level behavior of gap junction-coupled neuron systems consisting of class I cells, rather than the dynamics of isolated single cells. In this respect, we define a neuron class, say $\mathrm{I}^{*}$, in a more abstract setting, in which (a part of) the class I neurons of Connor type are included [4].

Let $I^{*}$ denote a subclass of class

I neurons, in which there is a certain interval J of $I$ (injected current) such that the following conditions hold simultaneously for $I$ in J:

1, there appears a narrow channel between $\mathrm{N}_{\mathrm{V}}$ and $\mathrm{N}_{\mathrm{R}}$,

2 , there is a unique intersection of $\mathrm{N}_{\mathrm{V}}$ and $\mathrm{N}_{\mathrm{R}}$ which is unstable spiral,

3 , there exist orbits (with positive measure) reentering into the channel.

We claim that class I* neurons, when coupled by gap junctions, exhibit extensive spatio-temporal chaos in some parameter regions [4]. Note that they show perfectly regular firings when isolated, hence this chaotic behavior is an emergent property of coupled systems.
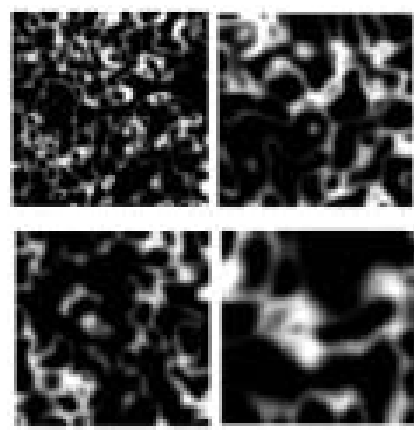
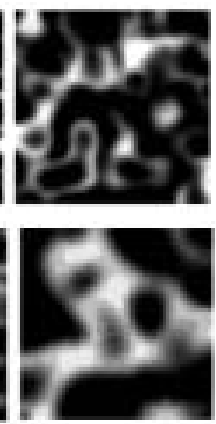

Fig. 2

Snapshots of contours of membrane potentials of GJ-coupled $80 x 80$ class I* neurons.

(a ) (top) 4-nearest neighbor couplings

(b) (below) 8-nearest neighbor couplings

The gap junction strength $g_{G J}$ is, from left to right, 0.5, 1.5 and 3.

In the following, we show some numerical results showing time series of membrane potentials of three neurons among 10 by 10 neurons coupled by gap junctions with nearest 4 neighbors (Fig.1). The injected current $I$ is constant both in time and space. The maximum Lyapunov exponent is positive. (Note: the gap junction couplings are assumed to be of linear diffusion type in this paper. See, [14].)

Fig.2 (a), (b) show snapshots of membrane potential contours made by $80 \times 80$ coupled neurons, with increasing gap junction conductance $\mathrm{g}_{\mathrm{GJ}}$. This shows how robust the emerged spatio-temporal chaos is; it appears in a wide range of parameters near the saddle-node bifurcation points, and even for far larger 
injection currents if the coupling $\mathrm{g}_{\mathrm{GJ}}$ is smaller.

\section{Chaotic itinerancy among quasi-attractors which transiently exhibit synchrony states}

Chaotic itinerancy is addressed as a transitory and sometimes nonstationary dynamics. (I.Tsuda [16]-[18]). The dynamical orbits once approach one of "quasi-attractors" (or, "attractor ruins") and stay for sometime with a certain distribution, and escape there and approach to other ruins. This transitory dynamics continues without external perturbations. In chaotic itinerancy, a "quasi-attractor" can be defined by the attractor in the sense of Milnor [9] in which both topological and measure-theoretic concepts play role. The main characteristics of chaotic itinerancy are: (1) the presence of many near-zero Lyapunov exponents, (2) slow decay of correlations/ (mutual) information, (3) history (path)-dependent transition, (4) no ergodicity, (5) nonconvergence or an extremely slow convergence of near-zero and/or even the largest Lyapunov exponents in some case [13], [18], (6) the lack of attractor-tracing property as well as the lack of pseudo-orbit tracing property [12]. In fact, we generally observe in our numerical data the dimension gap, i.e., the Lyapunov dimension (which may approximate Haussdorf dimension of the chaotic attractor) is bigger than the topological dimension by more than one. This dimension gap stems from a large number of negative Lyapunov exponents with a small absolute value. This brings about distributed attractors in phase space.

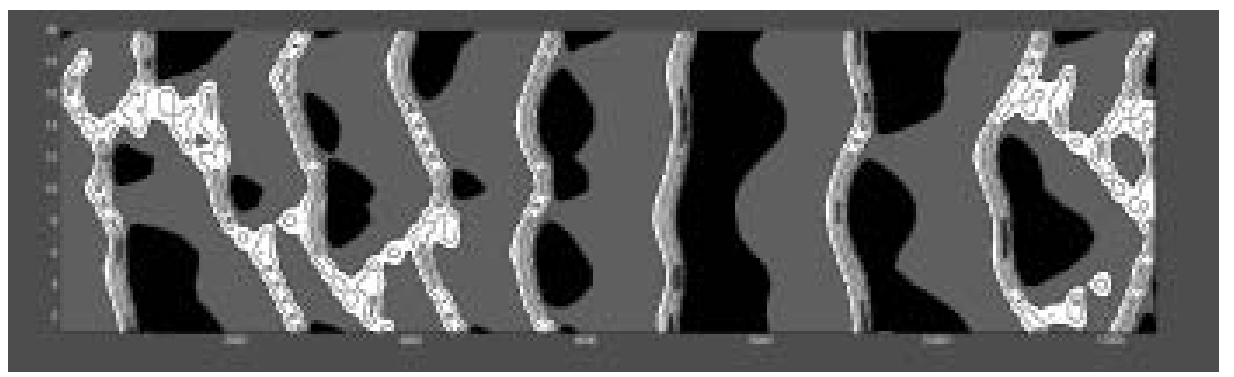

Fig. 3 Contour map of membrane potentials of 20 neurons of class I neurons coupled with the two neighbors by gap junctions. The vertical and horizontal directions indicate respectively the neuron positions, and the time $(0->250 \mathrm{msec})$. Each neuron receives a constant injected current without any external fluctuations. No structural irregularity exists in this model.

Figure 3 shows a contour map of time series of membrane potentials of the coupled class I* neurons. We observe metachronal waves which chaotically repeat creations and annihilations. (A metachronal wave is a wave produced by successive phase shifts of neighboring neurons' activity like the movement of centipedes or cilium and flagella.) If a metachronal wave with a short time lag can be viewed as a synchronized state, Figure 3 and other examples of our numerical results may indicate the presence of transient synchrony - chaotic alteration of synchronized and desynchronized states; the alternation is chaotic both in its 
timing and spatio-temporal patterns.

\section{Hypotheses - LFP fluctuations and its transient synchrony/binding problem}

We proposed in this paper a theoretical possibility that gap junction-coupled interneuron systems consisting of class $I^{*}$ neurons may emerge a chaotic itinerancy itinerant among quasi-attractors, characterized as cell groups which dynamically show transitions between synchronized and desynchronized states. Along the line of this view, we shall propose new hypotheses on the physiologically observed data of cortical dynamics, the concept of dynamical cell assembly and the so-called binding problem.

Our first proposal is expressed as the inhibitory chaotic field hypothesis, which says that the origin of the LFP fluctuations and stimulus-dependent transient synchrony, firstly observed and claimed by Gray [7] as the indicator of feature binding, is the itinerant chaos exhibited in gap junction-coupled interneuron systems consisting of class I* neurons. It should be noted, however, that chaotic fluctuations of inhibitory interneurons, say of FS cells in cortical layers, are not directly reflected in LFP. Rather, the inhibitory influence of FS cells to pyramidal cells affects the fluctuations of membrane potentials of the latter (pyramidal) cells, and the consequences of which may be observed as transitory and synchrony dynamics of LFP, as observed by Gray.

This may also lead us to another hypothesis that feature bindings may be established by joint itinerant dynamics of pyramidal and background FS cells.

Although many questions, including whether cortical FS cells belong to class I* (even in an approximate sense) and so on, remain open, we hope that our proposals may provide a new scope for the study of neocortical dynamics and its cognitive role.

Acknowledgements: The first author is supported by the Advanced and Innovational Research Program in Life Sciences from Ministry of Education, Culture, Sports, Science and Technology, the Japanese Government.

\section{§. References}

[1] B. Cauli et al., Molecular and physiological diversity of cortical nonpyramidal cells, J. Neurosci. (1997), 3894-3906.

[2] J. A. Connor et al., Neural repetitive firing: modifications of the Hodgkin-Huxley axon suggested by experimental results from crustacean axons, Biophysical Journal, 18 (1977) 81-102.

[3] H. Fujii et al., Dynamical cell assembly hypothesis -- Theoretical possibility of spatio-temporal coding in the cortex, Neural Networks 9 (1996) 1303-1350.

[4] H. Fujii and I. Tsuda, manuscript.

[5] M. Galarreta and S. Hestrin, A network of fast-spiking cells in the neocortex connected by electrical synapses, Nature, 402 (1999) 72-75.

[6] J.R. Gibson et al., Two networks of electrically coupled inhibitory neurons in neocortex. 
Nature 402 (1999), 75-79.

[7] C. Gray, et al., W., Synchronization of oscillatory neuronal responses in cat striate cortex: Temporal properties, Visual Neuroscience, 8 (1992) 337-347.

[8] A. L. Hodgkin, The local electric changes associated with repetitive action in a non-medullated axon, Journal of Physiology, 107 (1948) 165-181.

[9] J. Milnor, On the concept of attractor, Communications in Mathematical Physics, 99 (1985) 177-195.

[10] J. Rinzel, Excitation dynamics: insights from simplified membrane models, Fed. Proc., 44 (1985) 2944-2946.

[11] M. A. Rogawski. The A-current: how ubiquitous a feature of excitable cells is it? TINS 8 (1985), 214-219.

[12] R. M. Rose and J. L. Hindmarsh, The assembly of ionic currents in a thalamic neuron I. The three-dimensional model. Proceedings of Royal Soceity of London B 237 (1989) 267-288.

[13] T. Sauer, Abstracts for SIAM Pacific Rim Dynamical Systems Conference, August 9-13, 2000, Hawaii, 51; Chaotic itinerancy based on attractors of one-dimensional maps, Chaos 13 (2003) 947-952.

[14] N. Schweighofer et al., Electrophysiological Properties of Inferior Olive Neurons: A Compartment model, Journal of Neurophysiology, 82 (1999) 804-817.

[15] G. Tamas et al., Proximally targeted GABAergic synapses and gap junctions synchronize cortical interneurons, Neuroscience, 3 (2000) 366-371.

[16] I. Tsuda, Chaotic itinerancy as a dynamical basis of Hermeneutics of brain and mind. World Futures, 32 (1991) 167-185.

[17] I. Tsuda, Toward an interpretation of dynamic neural activity in terms of chaotic dynamical systems, Behavioral and Brain Sciences, 24 (2001) 793-847.

[18] I. Tsuda and T. Umemura, Chaotic itinerancy generated by coupling of Milnor attractors, Chaos 13 (2003),926-936.

[19] H. R. Wilson, Simplified dynamics of human and mammalian neocortical neurons, Journal Theoretical Biology, 200 (1999) 375-388.

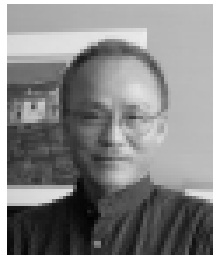

Hiroshi Fujii is Professor at the Department of Information and Communication Sciences, Kyoto Sangyo University. After receiving a Ph.D. from Kyoto University in 1973, his principal research area had been nonlinear mathematics, e.g., bifurcation theory and dynamical systems theory for nonlinear PDEs until 1989. He then turned his field to brain researches, and his main question has been how realistic cortical dynamics can carry cognitive functions in the real brain. He is presently a member of the project "Advanced and Innovational Research Program in Life Sciences" of the Japanese Government.

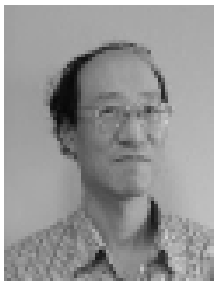

Ichiro Tsuda holds the post of Professor in the Department of Mathematics, Hokkaido University, as well as Visiting Professor at the School of Engineering, Osaka University. He is also Advisory Committee Member of Center for Complex Systems Study at Kalamazoo College. He was group leader of the Basic Design Group, National Bioholonics Project of ERATO in Japan. He has published widely in the field of chaotic dynamical systems and the brain. 